\title{
The Redox Properties of Ascorbate Peroxidase
}

Igor Efimov, ${ }^{\ddagger}$ Nektaria D. Papadopoulou, ${ }^{\ddagger}$ Kirsty J. McLean, ${ }^{\S}$ Sandip K Badyal ${ }^{\ddagger}$, Isabel K Macdonald, ${ }^{\ddagger}$ Andrew W. Munro, ${ }^{\S}$ Peter C. E. Moody? and Emma Lloyd Raven ${ }^{*},+$

Department of Chemistry, University of Leicester, University Road, Leicester, LE1 7RH, United Kingdom, Department of Biochemistry and Henry Wellcome Laboratories for Structural Biology, Henry Wellcome Building, University of Leicester, Lancaster Road, Leicester, LE1 9HN, United Kingdom, and Manchester Interdisciplinary Biocentre, Faculty of Life Sciences, University of Manchester, 131 Princess Street,

Manchester, M1 7DN, United Kingdom .

\section{SUPPLEMENTARY FIGURE}

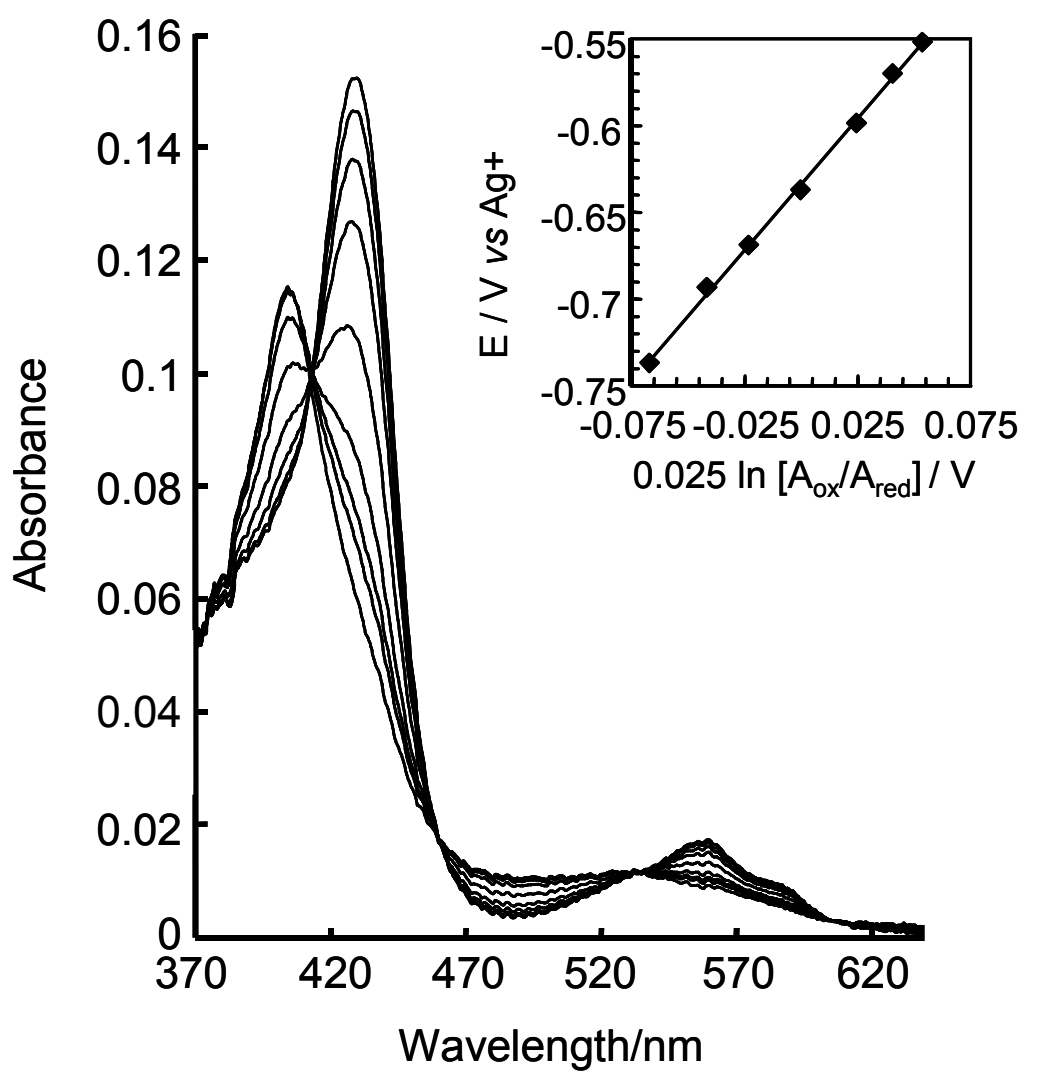

Figure S1. UV-vis spectra of W41A during reduction using an OTTLE cell. Inset: the corresponding Nernst plot for 1 electron transfer. 